$\begin{array}{ll}\text { Mathematical Analysis } & \text { Vol. } 1 \text { (2020), No. 1, 15-24 } \\ \text { \& Convex Optimization } & \text { https: } \backslash \backslash \text { maco.lu.ac.ir } \\ & \text { DOI: } 10.29252 / \text { maco.1.1.3 }\end{array}$

\author{
Research Paper
}

\title{
SCHUR-CONVEXITY OF INTEGRAL ARITHMETIC MEANS OF CO-ORDINATED CONVEX FUNCTIONS IN $\mathbb{R}^{3}$
}

\author{
NOZAR SAFAEI AND ALI BARANI*
}

\begin{abstract}
In this paper, we investigate Schur-convexity of some functions which are obtained from the co-ordinated convex functions on a rectangular box in $\mathbb{R}^{3}$
\end{abstract}

MSC(2010): 26A51; 26D15.

Keywords: Schur-convexity, Convex functions on the co-ordinates.

\section{Introduction}

The first study of Schur-convexity was done by Issai Schur in 1923. Since then numerous articles have been written about it, see for exmple [3, 4, 9, 10]. Schur-convexity has many important applications in analytic and geometric inequality, combinatorial analysis, combinatorial optimization, matrix theory, information theory, and other fields. We recall some definitions as follows:

Definition 1.1. [1] Suppose that $x=\left(x_{1}, x_{2}, \ldots, x_{n}\right), y=\left(y_{1}, y_{2}, \ldots, y_{n}\right) \in \mathbb{R}^{n} \cdot x$ is said to be majorized by $y$ (in symbols $x \prec y$ ) if

$$
\sum_{i=1}^{k} x_{[i]} \leq \sum_{i=1}^{k} y_{[i]} \quad k=1,2, \ldots, n-1
$$

and

$$
\sum_{i=1}^{n} x_{[i]}=\sum_{i=1}^{n} y_{[i]}
$$

where $x_{[i]}$, denotes the $\mathrm{i}$-th largest component in $x$.

Definition 1.2. [1] Let $E \subset \mathbb{R}^{n}, f: E \rightarrow \mathbb{R}$ is said to be Schur-convex function on $E$ if $x \prec y$ on $E$ implies $f(x) \leq f(y)$. $f$ is said to be Schur-concave if and only if $-f$ is Schur-convex.

Definition 1.3. [1,8] (i) A set $E \subset \mathbb{R}^{n}$ is called symmetric, if $x \in E$ implies $P x \in E$ for every $n \times n$ permutation matrix $P$.

(ii) A function $f: E \rightarrow \mathbb{R}$ is is said to be a symmetric function if $f(P x)=f(x)$ for every permutation matrix $P$, and for every $x \in E$.

Recall that a $n \times n$ square matrix $P$ is said to be a permutation matrix if each row and column has a single unite entry, and all other entries are zero. The following theorem called the schur's condition, is very useful for specifying Schur-convexity or Schur-concavity of functions.

Date: Received: October 24, 2019, Accepted: February 12, 2020 .

${ }^{*}$ Corresponding author. 
Theorem 1.4. [1] Let $E \subset \mathbb{R}^{n}$ be a symmetric convex set with nonempty interior ( $E^{\circ}$ is the interior of $E$ ), and $f: E \rightarrow \mathbb{R}$ is a symmetric continuous function on $E$. If $f$ is differentiable on $E^{\circ}$, then $f$ is Schur-convex (Schur-concave) on $E^{\circ}$ if and only if

$$
\left(x_{1}-x_{2}\right)\left(\frac{\partial f}{\partial x_{1}}-\frac{\partial f}{\partial x_{2}}\right) \geq 0 \quad(\leq 0)
$$

for every $x=\left(x_{1}, x_{2}, \ldots, x_{n}\right) \in E^{\circ}$.

In [5], S.S. Dragomir defined convex function on the co-ordinates (or co-ordinated convex functions ) on the set $[a, b] \times[c, d]$ in $\mathbb{R}^{2}$ with $a<b$ and $c<d$ as follows:

Definition 1.5. A function $f:[a, b] \times[c, d] \rightarrow \mathbb{R}$ is said to be convex on the co-ordinates on $[a, b] \times[c, d]$ if for every $y \in[c, d]$ and $x \in[a, b]$, the partial mappings,

$$
f_{y}:[a, b] \rightarrow \mathbb{R}, \quad f_{y}(u)=f(u, y)
$$

and

$$
f_{x}:[c, d] \rightarrow \mathbb{R}, \quad f_{x}(v)=f(x, v),
$$

are convex. This means that for every $(x, y),(z, w) \in[a, b] \times[c, d]$ and $t, s \in[0,1]$,

$$
\begin{aligned}
f(t x+(1-t) z, s y+(1-s) w) \leq & t s f(x, y)+s(1-t) f(z, y) \\
& +t(1-s) f(x, w)+(1-t)(1-s) f(z, w) .
\end{aligned}
$$

Clearly, every convex function is co-ordinated convex. Furthermore, there exist co-ordinated convex functions which are not convex. The following Hermite-Hadamard type inequality for co-ordinated convex functions was also proved in [5].

Theorem 1.6. Suppose that $f:[a, b] \times[c, d] \rightarrow \mathbb{R}$ is convex on the co-ordinates on $[a, b] \times[c, d]$. Then,

$$
\begin{aligned}
f\left(\frac{a+b}{2}, \frac{c+d}{2}\right) \leq & \frac{1}{2}\left[\frac{1}{b-a} \int_{a}^{b} f\left(x, \frac{c+d}{2}\right) d x+\frac{1}{d-c} \int_{c}^{d} f\left(\frac{a+b}{2}, y\right) d y\right] \\
\leq & \frac{1}{(b-a)(d-c)} \int_{a}^{b} \int_{c}^{d} f(x, y) d y d x \\
\leq & \frac{1}{4}\left[\frac{1}{b-a} \int_{a}^{b} f(x, c) d x+\frac{1}{b-a} \int_{a}^{b} f(x, d) d x\right. \\
& \left.+\frac{1}{d-c} \int_{c}^{d} f(a, y) d y+\frac{1}{d-c} \int_{c}^{d} f(b, y) d y\right] \\
\leq & \frac{f(a, c)+f(a, d)+f(b, c)+f(b, d)}{4} .
\end{aligned}
$$

The above inequalities are sharp.

In [7], M.E. Özdemir defined convex function on a rectangular box $\Omega=[a, b] \times[c, d] \times[e, f]$ in $\mathbb{R}^{3}$ as follows: A function $f: \Omega \rightarrow \mathbb{R}$ is said to be convex on the co-ordinates on $\Omega$ if for every $(x, y) \in[a, b] \times[c, d],(x, z) \in[a, b] \times[e, f]$ and $(y, z) \in[c, d] \times[e, f]$, the partial mappings,

$$
\begin{aligned}
& f_{z}:[a, b] \times[c, d] \rightarrow \mathbb{R}, \quad f_{z}(u, v)=f(u, v, z), \quad z \in[e, f], \\
& f_{y}:[a, b] \times[e, f] \rightarrow \mathbb{R}, \quad f_{y}(u, w)=f(u, y, w), \quad y \in[c, d], \\
& f_{x}:[c, d] \times[e, f] \rightarrow \mathbb{R}, \quad f_{x}(v, w)=f(x, v, w), \quad x \in[a, b],
\end{aligned}
$$

are convex. The following theorem is given in [7]. 
Theorem 1.7. Suppose that $f: \Omega=[a, b] \times[c, d] \times[e, f] \rightarrow \mathbb{R}$ is convex on the co-ordinates on $\Omega$. Then one has the inequalities:

$$
\begin{aligned}
f\left(\frac{a+b}{2}, \frac{c+d}{2}, \frac{e+f}{2}\right) \leq & \frac{1}{(b-a)(d-c)(f-e)} \iiint_{\Omega} f(x, y, z) d y d z d x \\
\leq & \frac{1}{6}\left[\frac{1}{(b-a)(d-c)} \iint_{\Delta_{1}} f(x, y, e) d y d x\right. \\
& +\frac{1}{(b-a)(d-c)} \iint_{\Delta_{1}} f(x, y, f) d y d x \\
& +\frac{1}{(b-a)(f-e)} \iint_{\Delta_{2}} f(x, c, z) d z d x \\
& +\frac{1}{(b-a)(f-e)} \iint_{\Delta_{2}} f(x, d, z) d z d x \\
& +\frac{1}{(d-c)(f-e)} \iint_{\Delta_{3}} f(a, y, z) d z d y \\
& \left.+\frac{1}{(d-c)(f-e)} \iint_{\Delta_{3}} f(b, y, z) d z d y\right]
\end{aligned}
$$

where $\Delta_{1}=[a, b] \times[c, d], \Delta_{2}=[a, b] \times[e, f]$ and $\Delta_{3}=[c, d] \times[e, f]$.

In [6] Elezović and Pečarić investigated the Schur-convexity on the upper and the lower limit of the integral for the mean of convex function and proved the following important result by using the Hermite-Hadamard inequality.

Theorem 1.8. Let $f$ be a continuous function on an interval $I$, and

$$
F(x, y)= \begin{cases}\frac{1}{y-x} \int_{x}^{y} f(t) d t, & x, y \in I, x \neq y, \\ f(x), & x=y \in I .\end{cases}
$$

Then $F(x, y)$ is Schur-convex (Schur-concave) on $I^{2}$ if and only if $f$ is convex (concave) on $I$.

Let $I \subset \mathbb{R}$ be an open interval and $f \in C^{2}(I)$. In [3] Y. Chu et al. proved the following theorem.

Theorem 1.9. Let $f: I \rightarrow \mathbb{R}$ be a continuous function. The function

$$
F(x, y)= \begin{cases}\frac{1}{y-x} \int_{x}^{y} f(t) d t-f\left(\frac{x+y}{2}\right), & x, y \in I, x \neq y, \\ 0, & x=y \in I,\end{cases}
$$

is Schur-convex (Schur-concave) on $I^{2}$ if and only if $f$ is convex (concave) on $I$.

We recall the following lemma from [2], which is known as Leibniz's Formula.

Lemma 1.10. Suppose that $f: \Delta=[a, b] \times[c, d] \rightarrow \mathbb{R}$ and $\frac{\partial f}{\partial t}:[a, b] \times[c, d] \rightarrow \mathbb{R}$ are continuous and $\alpha_{1}, \alpha_{2}:[c, d] \rightarrow[a, b]$ are differentiable functions. Then, the function $\varphi:[c, d] \rightarrow \mathbb{R}$ defined by

$$
\varphi(t)=\int_{\alpha_{1}(t)}^{\alpha_{2}(t)} f(x, t) d x
$$


has a derivative for each $t \in[c, d]$, which is given by

$$
\varphi^{\prime}(t)=f\left(\alpha_{2}(t), t\right) \alpha_{2}^{\prime}(t)-f\left(\alpha_{1}(t), t\right) \alpha_{1}^{\prime}(t)+\int_{\alpha_{1}(t)}^{\alpha_{2}(t)} \frac{\partial f}{\partial t}(x, t) d x .
$$

Moreover, we use the following lemma which will be useful in the sequal. A version of the following lemma proved in [9].

Lemma 1.11. Let $F(u, v)=\int_{u}^{v} \int_{u}^{v} \int_{u}^{v} f(x, y, z) d x d y d z$, where

$$
f(x, y, z), \quad \frac{\partial}{\partial b} \int_{u}^{v} f(x, y, z) d x
$$

and

$$
\frac{\partial}{\partial b} \int_{u}^{v} \int_{u}^{v} f(x, y, z) d x d y
$$

are continuous on the cube $\Omega=[a, p] \times[a, p] \times[a, p], u=u(b)$ and $v=v(b)$ are differentiable with $a \leq u(b) \leq p$ and $a \leq v(b) \leq p$. Then,

$$
\begin{aligned}
\frac{\partial F}{\partial b}= & \left(\int_{u}^{v} \int_{u}^{v} f(x, y, v) d x d y+\int_{u}^{v} \int_{u}^{v} f(x, v, z) d x d z\right. \\
& \left.+\int_{u}^{v} \int_{u}^{v} f(v, y, z) d y d z\right) v^{\prime}(b)-\left(\int_{u}^{v} \int_{u}^{v} f(x, y, u) d x d y\right. \\
& \left.+\int_{u}^{v} \int_{u}^{v} f(x, u, z) d x d z+\int_{u}^{v} \int_{u}^{v} f(u, y, z) d y d z\right) u^{\prime}(b) .
\end{aligned}
$$

Proof. If $G(u, v, y, z)=\int_{u}^{v} f(x, y, z) d x$ and $H(u, v, z)=\int_{u}^{v} G(u, v, y, z) d y$ then $F(u, v)=$ $\int_{u}^{v} H(u, v, z) d z$. Therefore by Lemma 1.10, we have

$$
\begin{gathered}
\frac{\partial F}{\partial b}=H(u, v, v) v^{\prime}(b)-H(u, v, u) u^{\prime}(b)+\int_{u}^{v} \frac{\partial H(u, v, z)}{\partial b} d z \\
\frac{\partial H(u, v, z)}{\partial b}=G(u, v, v, z) v^{\prime}(b)-G(u, v, u, z) u^{\prime}(b) \\
+\int_{u}^{v} \frac{\partial G(u, v, y, z)}{\partial b} d y, \\
\frac{\partial G(u, v, y, z)}{\partial b}=f(v, y, z) v^{\prime}(b)-f(u, y, z) u^{\prime}(b) .
\end{gathered}
$$

By replacing (1.3) and (1.4) in (1.2) we obtained required result in (1.1).

\section{Main Results}

In this section we prove new theorems like those Theorem 1.8 and Theorem 1.9 for coordinated convex functions.

To reach our main results, we need the following two lemmas.

Lemma 2.1. Let $\Omega:=\left[a_{1}, b_{1}\right] \times\left[a_{1}, b_{1}\right] \times\left[a_{1}, b_{1}\right]$ be a cube in $\mathbb{R}^{3}$ with $a_{1}<b_{1}$, and the function $f: \Omega \rightarrow \mathbb{R}$ is continuous, and has continuous second order partial derivatives on $\Omega^{\circ}$ ( 
the interior of $\Omega)$. Choose $a, b \in\left(a_{1}, b_{1}\right)$, with $a<b$, and let $D:=[a, b] \times[a, b]$. Suppose that the function $F: D \rightarrow \mathbb{R}$ is defined by

$$
F(x, y):=\left\{\begin{array}{lll}
\frac{1}{(y-x)^{3}} \int_{x}^{y} \int_{x}^{y} \int_{x}^{y} f(r, s, t) d r d s d t, & x \neq y, & x, y \in[a, b], \\
f(x, x, x), & x=y, & x, y \in[a, b] .
\end{array}\right.
$$

Then,

$$
\begin{aligned}
\left.\frac{\partial F}{\partial x}\right|_{\left(t_{0}, t_{0}\right)}= & \left.\frac{\partial F}{\partial y}\right|_{\left(t_{0}, t_{0}\right)} \\
= & \frac{1}{24}\left[\left.6 \frac{\partial f}{\partial t}(t, t, t)\right|_{t_{0}}+2\left(g_{1}\left(t_{0}, t_{0}, t_{0}\right)+g_{2}\left(t_{0}, t_{0}, t_{0}\right)+g_{3}\left(t_{0}, t_{0}, t_{0}\right)\right.\right. \\
& \left.\left.+f_{1}\left(t_{0}, t_{0}, t_{0}\right)+f_{2}\left(t_{0}, t_{0}, t_{0}\right)+f_{3}\left(t_{0}, t_{0}, t_{0}\right)\right)\right]
\end{aligned}
$$

for all $t_{0} \in[a, b]$, where

$$
\begin{aligned}
f_{1}\left(u, v, t_{0}+t\right) & =\frac{\partial f}{\partial t}\left(u, v, t_{0}+t\right), \\
f_{2}\left(u, t_{0}+t, w\right) & =\frac{\partial f}{\partial t}\left(u, t_{0}+t, w\right), \\
f_{3}\left(t_{0}+t, v, w\right) & =\frac{\partial f}{\partial t}\left(t_{0}+t, v, w\right),
\end{aligned}
$$

and

$$
\begin{aligned}
g_{1}\left(u, t_{0}+t, t_{0}+t\right) & =\frac{\partial f}{\partial t}\left(u, t_{0}+t, t_{0}+t\right), \\
g_{2}\left(t_{0}+t, v, t_{0}+t\right) & =\frac{\partial f}{\partial t}\left(t_{0}+t, v, t_{0}+t\right), \\
g_{3}\left(t_{0}+t, t_{0}+t, w\right) & =\frac{\partial f}{\partial t}\left(t_{0}+t, t_{0}+t, w\right) .
\end{aligned}
$$

Proof. Fix $t_{0} \in[a, b]$. We put

$$
\begin{aligned}
h_{1}\left(u, v, t_{0}+t\right) & =\frac{\partial f_{1}}{\partial t}\left(u, v, t_{0}+t\right), \\
h_{2}\left(u, t_{0}+t, w\right) & =\frac{\partial f_{2}}{\partial t}\left(u, t_{0}+t, w\right), \\
h_{3}\left(t_{0}+t, v, w\right) & =\frac{\partial f_{3}}{\partial t}\left(t_{0}+t, v, w\right) .
\end{aligned}
$$

By using the L'Hopital's rule, and Lemmas 1.10, 1.11 we see that

$$
\begin{aligned}
\left.\frac{\partial F}{\partial x}\right|_{\left(t_{0}, t_{0}\right)}= & \lim _{t \rightarrow 0} \frac{F\left(t_{0}+t, t_{0}\right)-F\left(t_{0}, t_{0}\right)}{t} \\
= & \lim _{t \rightarrow 0} \frac{1}{t^{4}}\left[\int_{t_{0}}^{t_{0}+t} \int_{t_{0}}^{t_{0}+t} \int_{t_{0}}^{t_{0}+t} f(u, v, w) d u d v d w-t^{3} f\left(t_{0}, t_{0}\right)\right] \\
= & \lim _{t \rightarrow 0} \frac{1}{4 t^{3}}\left[\int_{t_{0}}^{t_{0}+t} \int_{t_{0}}^{t_{0}+t} f\left(u, v, t_{0}+t\right) d u d v+\int_{t_{0}}^{t_{0}+t} \int_{t_{0}}^{t_{0}+t} f\left(u, t_{0}+t, w\right) d u d w\right. \\
& \left.+\int_{t_{0}}^{t_{0}+t} \int_{t_{0}}^{t_{0}+t} f\left(t_{0}+t, v, w\right) d v d w-3 t^{2} f\left(t_{0}, t_{0}, t_{0}\right)\right]
\end{aligned}
$$




$$
\begin{aligned}
= & \lim _{t \rightarrow 0} \frac{1}{12 t^{2}}\left[\int_{t_{0}}^{t_{0}+t} f\left(u, t_{0}+t, t_{0}+t\right) d u+\int_{t_{0}}^{t_{0}+t} f\left(t_{0}+t, v, t_{0}+t\right) d v\right. \\
& +\int_{t_{0}}^{t_{0}+t} \int_{t_{0}}^{t_{0}+t} \frac{\partial f}{\partial t}\left(u, v, t_{0}+t\right) d u d v+\int_{t_{0}}^{t_{0}+t} f\left(u, t_{0}+t, t_{0}+t\right) d u \\
& +\int_{t_{0}}^{t_{0}+t} f\left(t_{0}+t, t_{0}+t, w\right) d w+\int_{t_{0}}^{t_{0}+t} \int_{t_{0}}^{t_{0}+t} \frac{\partial f}{\partial t}\left(u, t_{0}+t, w\right) d u d w \\
& +\int_{t_{0}}^{t_{0}+t} f\left(t_{0}+t, v, t_{0}+t\right) d v+\int_{t_{0}}^{t_{0}+t} f\left(t_{0}+t, t_{0}+t, w\right) d w \\
& \left.+\int_{t_{0}}^{t_{0}+t} \int_{t_{0}}^{t_{0}+t} \frac{\partial f}{\partial t}\left(t_{0}+t, v, w\right) d v d w-6 t f\left(t_{0}, t_{0}, t_{0}\right)\right] \\
= & \lim _{t \rightarrow 0} \frac{1}{24 t}\left[6 f\left(t_{0}+t, t_{0}+t, t_{0}+t\right)+2 \int_{t_{0}}^{t_{0}+t} \frac{\partial f}{\partial t}\left(u, t_{0}+t, t_{0}+t\right) d u\right. \\
& +2 \int_{t_{0}}^{t_{0}+t} \frac{\partial f}{\partial t}\left(t_{0}+t, v, t_{0}+t\right) d v+2 \int_{t_{0}}^{t_{0}+t} \frac{\partial f}{\partial t}\left(t_{0}+t, t_{0}+t, w\right) d w \\
& +\int_{t_{0}}^{t_{0}+t} f_{1}\left(u, t_{0}+t, t_{0}+t\right) d u+\int_{t_{0}}^{t_{0}+t} f_{1}\left(t_{0}+t, v, t_{0}+t\right) d v \\
& +\int_{t_{0}}^{t_{0}+t} \int_{t_{0}}^{t_{0}+t} h_{1}\left(u, v, t_{0}+t\right) d u d v+\int_{t_{0}}^{t_{0}+t} f_{2}\left(u, t_{0}+t, t_{0}+t\right) d u \\
& +\int_{t_{0}}^{t_{0}+t} f_{2}\left(t_{0}+t, t_{0}+t, w\right) d w+\int_{t_{0}}^{t_{0}+t} \int_{t_{0}}^{t_{0}+t} h_{2}\left(u, t_{0}+t, w\right) d u d w \\
& +\int_{t_{0}}^{t_{0}+t} f_{3}\left(t_{0} \frac{\partial f}{\partial t}(t, t, t) f_{t_{0}}+2\left(t_{0}+t, t_{0}+t, w\right) d w\right. \\
& \left.+\int_{t_{0}}^{t_{0}+t} \int_{t_{0}}^{t_{0}+t} h_{3}\left(t_{0}+t, v, w\right) d v d w-6 f\left(t_{0}, t_{0}, t_{0}\right)\right] \\
& \left.\left.\left.\left.f_{0}, t_{0}, t_{0}\right)+t_{0}\right)+g_{2}\left(t_{0}, t_{0}, t_{0}\right)+t_{0}, t_{0}, t_{0}\right)+f_{0}, t_{0}, t_{0}\right) \\
& \left.\left.=t_{0}, t_{0}, t_{0}\right)\right] . \\
&
\end{aligned}
$$

By changing the role of $x$ by $y$ in (2.2), we obtain required results in (2.1).

The proof of the following lemma is similar to once in lemma 2.1 hence we omit it.

Lemma 2.2. Let $\Omega:=\left[a_{1}, b_{1}\right] \times\left[a_{1}, b_{1}\right] \times\left[a_{1}, b_{1}\right]$ be a cube in $\mathbb{R}^{3}$ with $a_{1}<b_{1}$, and the function $f: \Omega \rightarrow \mathbb{R}$ is continuous, and has continuous four order partial derivatives on $\Omega^{\circ}$. Choose 
$a, b \in\left(a_{1}, b_{1}\right)$, with $a<b$, and let $D:=[a, b] \times[a, b]$. Suppose that the function $G: D \rightarrow \mathbb{R}$ is defined by

$$
G(x, y):= \begin{cases}\frac{1}{(y-x)^{3}} \int_{x}^{y} \int_{x}^{y} \int_{x}^{y} f(r, s, t) d r d s d t & \\ -f\left(\frac{x+y}{2}, \frac{x+y}{2}, \frac{x+y}{2}\right), & x \neq y, x, y \in[a, b] \\ 0, & x=y, x, y \in[a, b] .\end{cases}
$$

Then,

$$
\begin{aligned}
\left.\frac{\partial G}{\partial x}\right|_{\left(t_{0}, t_{0}\right)}= & \left.\frac{\partial G}{\partial y}\right|_{\left(t_{0}, t_{0}\right)} \\
= & \frac{1}{24}\left[-\left.3 \frac{\partial f}{\partial t}(t, t, t)\right|_{t_{0}}+2\left(g_{1}\left(t_{0}, t_{0}, t_{0}\right)+g_{2}\left(t_{0}, t_{0}, t_{0}\right)+g_{3}\left(t_{0}, t_{0}, t_{0}\right)\right.\right. \\
& \left.+f_{1}\left(t_{0}, t_{0}, t_{0}\right)+f_{2}\left(t_{0}, t_{0}, t_{0}\right)+f_{3}\left(t_{0}, t_{0}, t_{0}\right)\right]
\end{aligned}
$$

for all $t_{0} \in[a, b]$, where

$$
\begin{aligned}
f_{1}\left(u, v, t_{0}+t\right) & =\frac{\partial f}{\partial t}\left(u, v, t_{0}+t\right), \\
f_{2}\left(u, t_{0}+t, w\right) & =\frac{\partial f}{\partial t}\left(u, t_{0}+t, w\right), \\
f_{3}\left(t_{0}+t, v, w\right) & =\frac{\partial f}{\partial t}\left(t_{0}+t, v, w\right),
\end{aligned}
$$

and

$$
\begin{aligned}
g_{1}\left(u, t_{0}+t, t_{0}+t\right) & =\frac{\partial f}{\partial t}\left(u, t_{0}+t, t_{0}+t\right), \\
g_{2}\left(t_{0}+t, v, t_{0}+t\right) & =\frac{\partial f}{\partial t}\left(t_{0}+t, v, t_{0}+t\right), \\
g_{3}\left(t_{0}+t, t_{0}+t, w\right) & =\frac{\partial f}{\partial t}\left(t_{0}+t, t_{0}+t, w\right) .
\end{aligned}
$$

We now derive the next results for co-ordinates convex functions.

Theorem 2.3. Let $D:=\left[a_{1}, b_{1}\right] \times\left[a_{1}, b_{1}\right] \times\left[a_{1}, b_{1}\right]$ be a cube in $\mathbb{R}^{3}$ with $a_{1}<b_{1}$, and the function $f: D \rightarrow \mathbb{R}$ is continuous, and has continuous second order partial derivatives on $D^{\circ}$. Choose $a, b \in\left(a_{1}, b_{1}\right)$, with $a<b$, and let $\Delta:=[a, b] \times[a, b] \times[a, b]$. Suppose that $f$ is convex on the co-ordinates on $\Delta$, then the function $F:[a, b] \times[a, b] \rightarrow \mathbb{R}$ defined by

$$
F(x, y):=\left\{\begin{array}{lll}
\frac{1}{(y-x)^{3}} \int_{x}^{y} \int_{x}^{y} \int_{x}^{y} f(r, s, t) d r d s d t, & x \neq y, & x, y \in[a, b], \\
f(x, x, x), & x=y, & x, y \in[a, b] .
\end{array}\right.
$$

is Schur-convex on $[a, b] \times[a, b]$.

Proof. Case 1: If $x, y \in[a, b]$, with $x=y$. Then Lemma 2.1 implies that

$$
(y-x)\left(\frac{\partial F}{\partial y}-\frac{\partial F}{\partial x}\right)=0 .
$$


Case 2: If $x, y \in[a, b]$, with $x \neq y$. Then by Lemma 1.11 we have

$$
\begin{aligned}
\frac{\partial F}{\partial y}= & \frac{-3}{(y-x)^{4}} \int_{x}^{y} \int_{x}^{y} \int_{x}^{y} f(r, s, t) d r d s d t \\
& +\frac{1}{(y-x)^{3}}\left[\int_{x}^{y} \int_{x}^{y} f(r, s, y) d r d s\right. \\
& \left.+\int_{x}^{y} \int_{x}^{y} f(r, y, t) d r d t+\int_{x}^{y} \int_{x}^{y} f(y, s, t) d s d t\right]
\end{aligned}
$$

and

$$
\begin{aligned}
\frac{\partial F}{\partial x}= & \frac{3}{(y-x)^{4}} \int_{x}^{y} \int_{x}^{y} \int_{x}^{y} f(r, s, t) d r d s d t \\
& -\frac{1}{(y-x)^{3}}\left[\int_{x}^{y} \int_{x}^{y} f(r, s, x) d r d s\right. \\
& \left.+\int_{x}^{y} \int_{x}^{y} f(r, x, t) d r d t+\int_{x}^{y} \int_{x}^{y} f(x, s, t) d s d t\right] .
\end{aligned}
$$

Thus,

$$
\begin{aligned}
\left(\frac{\partial F}{\partial y}-\frac{\partial F}{\partial x}\right)= & \frac{-6}{(y-x)^{4}} \int_{x}^{y} \int_{x}^{y} \int_{x}^{y} f(r, s, t) d r d s d t \\
& +\frac{1}{(y-x)^{3}}\left[\int_{x}^{y} \int_{x}^{y}(f(r, s, x)+f(r, s, y)) d r d s\right. \\
& +\int_{x}^{y} \int_{x}^{y}(f(r, x, t)+f(r, y, t)) d r d t \\
& \left.+\int_{x}^{y} \int_{x}^{y}(f(x, s, t)+f(y, s, t)) d s d t\right] .
\end{aligned}
$$

Then $(y-x)\left(\frac{\partial F}{\partial y}-\frac{\partial F}{\partial x}\right)$ is nonnegative if

$$
\begin{aligned}
& \frac{1}{y-x} \int_{x}^{y} \int_{x}^{y} \int_{x}^{y} f(r, s, t) d r d s d t \\
\leq & \frac{1}{6}\left[\int_{x}^{y} \int_{x}^{y}(f(r, s, x)+f(r, s, y)) d r d s\right. \\
& +\int_{x}^{y} \int_{x}^{y}(f(r, x, t)+f(r, y, t)) d r d t \\
& \left.+\int_{x}^{y} \int_{x}^{y}(f(x, s, t)+f(y, s, t)) d s d t\right] .
\end{aligned}
$$

Since $f$ is convex on the co-ordinates the last inequality holds by Theorem 1.7. Therefore by Theorem 1.4 the function $F$ is Schur-convex.

The following theorem also holds:

Theorem 2.4. Let $D:=\left[a_{1}, b_{1}\right] \times\left[a_{1}, b_{1}\right] \times\left[a_{1}, b_{1}\right]$ be a cube in $\mathbb{R}^{3}$ with $a_{1}<b_{1}$, and the function $f: D \rightarrow \mathbb{R}$ is continuous, and has continuous four order partial derivatives on $D^{\circ}$. Choose $a, b \in\left(a_{1}, b_{1}\right)$, with $a<b$, and let $\Delta:=[a, b] \times[a, b] \times[a, b]$. Suppose that $f$ is convex 
on the co-ordinates on $\Delta$, then the function $G:[a, b] \times[a, b] \rightarrow \mathbb{R}$ defined by

$$
G(x, y):=\left\{\begin{array}{lll}
\frac{1}{(y-x)^{3}} \int_{x}^{y} \int_{x}^{y} \int_{x}^{y} f(r, s, t) d r d s d t & \\
-f\left(\frac{x+y}{2}, \frac{x+y}{2}, \frac{x+y}{2}\right), & x \neq y, & x, y \in[a, b], \\
0, & x=y, & x, y \in[a, b] .
\end{array}\right.
$$

is Schur-convex on $[a, b] \times[a, b]$.

Proof. Case 1: If $x, y \in[a, b]$, with $x=y$. Then Lemma 2.2 implies that

$$
(y-x)\left(\frac{\partial G}{\partial y}-\frac{\partial G}{\partial x}\right)=0
$$

Case 2: If $x, y \in[a, b]$, with $x \neq y$. Then by Lemma 1.11 we have

if

$$
(y-x)\left(\frac{\partial G}{\partial y}-\frac{\partial G}{\partial x}\right) \geq 0
$$

西

$$
\begin{aligned}
& \frac{1}{y-x} \int_{x}^{y} \int_{x}^{y} \int_{x}^{y} f(r, s, t) d r d s d t \\
\leq & \frac{1}{6}\left[\int_{x}^{y} \int_{x}^{y}(f(r, s, x)+f(r, s, y)) d r d s\right. \\
& +\int_{x}^{y} \int_{x}^{y}(f(r, x, t)+f(r, y, t)) d r d t \\
& \left.+\int_{x}^{y} \int_{x}^{y}(f(x, s, t)+f(y, s, t)) d s d t\right] .
\end{aligned}
$$

The result follows from Theorem 1.7 and Theorem 1.4.

In the following examples we show that the converses of theorems 2.3 and 2.4 are not true in general.

Example 2.5. Consider the non co-ordinates convex function,

$$
f(r, s, t):=r^{2}-\frac{1}{2} s^{2}+t^{2}, \quad r, t, s \in[1,2] .
$$

It is easy to see that for the function $F$ was defined in (2.3) we have $F(x, x)=\frac{3}{2} x^{2}$, for every $x \in[1,2]$, and

$$
F(x, y)=\frac{1}{(y-x)^{3}} \int_{x}^{y} \int_{x}^{y} \int_{x}^{y}\left(r^{2}-\frac{1}{2} s^{2}+t^{2}\right) d r d s d t=\frac{1}{2}\left(x^{2}+y^{2}+x y\right)
$$

for every $x, y \in[1,2]$, with $x \neq y$. Thus,

$$
F(x, y)=\frac{1}{2}\left(x^{2}+y^{2}+x y\right)
$$

for every $x, y \in[1,2]$. Clearly $F$ is symmetric, continuous and differentiable on $[1,2] \times[1,2]$. If $x, y \in[1,2]$, we have

$$
(y-x)\left(\frac{\partial F}{\partial y}-\frac{\partial F}{\partial x}\right)=\frac{1}{2}(y-x)^{2} \geq 0 .
$$

Therefore by Theorem $1.4 F$ is Schur-convex. 
Remark 2.6. It is easy to see that for the function $f$ was defined in example 2.5 each of the inequalities in theorem 1.7 is valid while $f$ is not convex on the co-ordintes. This means that the converse of theorem 1.7 is not valid in general.

Example 2.7. Consider the non co-ordinated convex function:

$$
f(r, s, t):=2 r^{2}-s^{2}+t^{2}, \quad r, t, s \in[1,2] .
$$

It is easy to see that for the function $G$ was defined in (2.4) we have $G(x, x)=0$, for every $x \in[1,2]$, and

$$
\begin{aligned}
G(x, y) & =\frac{1}{(y-x)^{3}} \int_{x}^{y} \int_{x}^{y} \int_{x}^{y}\left(2 r^{2}-s^{2}+t^{2}\right) d r d s d t-\frac{(x+y)^{2}}{2} \\
& =\frac{2}{3}\left(x^{2}+y^{2}+x y\right)-\frac{(x+y)^{2}}{2},
\end{aligned}
$$

for every $x, y \in[1,2]$, with $x \neq y$. Thus,

$$
G(x, y)=\frac{2}{3}\left(x^{2}+y^{2}+x y\right)-\frac{(x+y)^{2}}{2} .
$$

Clearly $G$ is symmetric, continuous and differentiable on $[1,2] \times[1,2]$. If $x, y \in[1,2]$, we have

$$
(y-x)\left(\frac{\partial G}{\partial y}-\frac{\partial G}{\partial x}\right)=\frac{2}{3}(y-x)^{2} \geq 0 .
$$

Therefore by Theorem 1.4 the function $G$ is Schur-convex.

\section{REFERENCES}

[1] B.C. Arnold, A.W. Marshal and I. Olkin, Inequalities : Theory of Majorization and It's Applications, Springer Series in Statistics, New York-Dordrecht-Heidelberg-London, 2011.

[2] R.G. Bartel, The Elements of Real Analysis, Jon Wiley and Sons, 2st edition, 1976.

[3] Y. Chu, G. Wang, and X. Zhang, Schur convexity and Hadamard's inequality. Mathematical Inequalities and Applications, 13 (4): 725-731, 2010.

[4] V. Culjak, I. Franjic, R. Ghulam, and J. Pečarić, Schur-Convexity of averages of convex functions. Journal of Inequalities and Applications, 25 pages, Article ID 581918, 2011.

[5] S.S. Dragomir, On the Hadamard's inequality for convex functions on the co-ordinates in a rectangle from the plan. Taiwanese Journal of Mathematics, 5: 775-778, 2001.

[6] N. Elezović and J. Pečarić, A note on Schur-convex functions. Rocky mountain Journal of Mathematics, 30 (3): 853-856, 2000.

[7] M.E. Özdemir and A.O. Akdemir, On some Hadamard-type inequalities for convex functions on a rectangular box. Journal of Nonlinear Analysis and Application, Volume 2011 (2011), Article ID jnaa-00101, 10 Pages doi: 10.5899/2011/jnaa-00101.

[8] A.W. Roberts and D.E. Varberg, Convex functions, Academic Press, New York, 1973.

[9] H.-N. Shi, Schur-convex functions related to Hadamard-type inequalities. Journal of Mathematical Inequalities, 1 (1): 127-136, 2007.

[10] X. Zhang and Y. Chu, Convexity of the integral arithmetic mean of a convex function. Rocky mountain Journal of Mathematics, 40 (3): 1061-1068, 2010.

(Nozar Safaei) Department of Mathematics, Lorestan University, Khorramabad, 6815144316, IRAN.

Email address: safaei.no@fs.lu.ac.ir

(Ali Barani) Department of Mathematics, Lorestan University, Khorramabad, 6815144316, IRAN.

Email address: barani.a@lu.ac.ir 